\title{
Reliability of Physiological Responses Induced by Basic Emotions: A Pilot Study
}

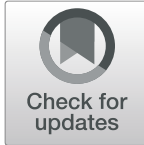

Eun-Hye Jang ${ }^{1}$, Sangwon Byun ${ }^{2^{*}+}$, Mi-Sook Park ${ }^{3}$ and Jin-Hun Sohn ${ }^{4^{*}+}$

\begin{abstract}
Background: Although emotion-specific autonomic responses based on the discrete theory of emotion have been widely studied, studies on the reliability of physiological responses to emotional stimuli are limited. In this study, we aimed to assess the reliability of physiological changes induced by the six basic emotions (happiness, sadness, anger, fear, disgust, and surprise) that were measured during 10 weekly repeated experiments.

Methods: Twelve college students participated, and in each experiment, physiological signals were collected before and while participants were watching emotion-provoking film clips. Additionally, the participants self-evaluated the emotions that they experienced during the film presentation at the end of each emotional stimulus. To avoid adaptation of participants to identical stimuli during repeated measurements, we used 10 different film clips for each emotion, and thus a total of 60 film clips over 10 weeks were used. Physiological features, such as skin conductance level $(\mathrm{SCL})$, fingertip temperature (FT), heart rate (HR), and blood volume pulse (BVP), were extracted from the physiological signals. Two reliability indices, Cronbach's alpha and intraclass correlation coefficient, were calculated from the physiological features to assess internal consistency and interrater reliability, respectively.

Results: We found that $\mathrm{SCL}, \mathrm{HR}$, and BVP measured during the emotion-provoking phase over the 10 weekly sessions were more reliable than those assessed at baseline. Furthermore, SCL, HR, and BVP from the emotionprovoking phase exhibited excellent internal consistency and interrater reliability.

Conclusions: Our findings suggest that these features can be used as reliable physiological indices in emotion studies. The results also support the significance of physiological signals as meaningful indicators for emotion recognition in $\mathrm{HCl}$ (human computer interface) area.
\end{abstract}

Keywords: Emotion, Physiological responses, Consistency, Reliability, Autonomic nervous system

\section{Background}

Physiological and behavioral changes are fundamental aspects of emotions, and emotions therefore cannot be simply interpreted as "feelings" or mental states [1]. Autonomic responses in emotions have been an active research topic since Cannon [2] first reported the physiology of emotions [3, 4]. Autonomic nervous system (ANS) activity has been viewed as a major component of emotion response in many recent theories of emotion [4]. Cacioppo and colleagues [5, 6] reported reliable

\footnotetext{
* Correspondence: swbyun@inu.ac.kr; jhsohn@cnu.ac.kr

†Sangwon Byun and Jin-Hun Sohn contributed equally to this work.

${ }^{2}$ Department of Electronics Engineering, Incheon National University, 119

Academy-ro, Yeonsu-gu, Incheon, Republic of Korea

${ }^{4}$ Department of Psychology, Brain Research Institute, Chungnam National

University, 99 Daehak-ro, Yuseong-gu, Daejeon 34134, Republic of Korea

Full list of author information is available at the end of the article
}

differences in autonomic responses between specific emotions, that is, autonomic emotion specificity. They also noted context-specific effects of ANS activity in emotion, which were related to different induction paradigms $[5,6]$. Furthermore, ANS responses exhibited more consistent valence-specific patterns than emotionspecific patterns: negative emotions were accompanied by stronger autonomic responses than positive emotions [4].

As emotion-specific ANS responses have been widely studied (see Kreibig's review article [4]), previous studies have attempted to assess the reliability of physiological responses induced by basic emotions [7]. For example, the temporal stability of physiological responses [8-13] and the reliability of event-related desynchronization in EEGs [14, 15] have been studied. Typically, intervals of

(c) The Author(s). 2019 Open Access This article is distributed under the terms of the Creative Commons Attribution 4.0 International License (http://creativecommons.org/licenses/by/4.0/), which permits unrestricted use, distribution, and 
2-4 weeks were introduced between test-retest experiments to measure the stability and consistency of physiological responses [16-19]. Various other biomarkers, including blinking responses, respiratory sinus arrhythmia, heartbeat period, salivary cortisol, and startle response, have also been tested [16-19]. However, most of these studies relied on a single repeat (test-retest) and used an identical stimulus for emotion elicitation, which may complicate the interpretation of results. Repeated measurements with the same stimulus may be affected by adaptation of participants to stimuli or learning effects (e.g., habituation). Furthermore, consistency over a relatively long period has not been evaluated.

In most studies on autonomic specificity of basic emotions, the direction of changes in ANS activity was evaluated as the difference between the baseline measurement and emotional condition [4], in which the baseline activity was used as a reference for observing state change in psychophysiological responses [20]. Since the quality of the baseline data against which autonomic change is evaluated relies on the methodology, previous studies have developed baseline calibration techniques for establishing an appropriate baseline, which would enable reliable assessment of psychophysiological changes during emotional state [21]. However, intra- and interindividual variations in the baseline level have not been studied separately. In particular, intraindividual variations in the baseline have been implicitly assumed to be not substantial.

Therefore, we aimed to examine the consistency of physiological responses that are measured multiple times over a relatively long period, using non-identical stimuli. We evaluated skin conductance level (SCL) and heart rate (HR), which are the most frequently reported measures of autonomic function [14]. We also measured fingertip temperature (FT) [22] and blood volume pulse (BVP) $[23,24]$ as additional indicators of the ANS activity. Based on these physiological measurements, we evaluated the consistency of baseline activity and autonomic responses related to six basic emotions (happiness, sadness, anger, fear, disgust, and surprise) during 10 weekly sessions. We hypothesized that physiological responses would show high consistency in both the baseline and emotional phases.

\section{Methods}

\section{Participants}

Twelve healthy subjects between 19 and 23 years of age ( 6 males and 6 females, mean age \pm SD, $21.0 \pm 1.98$ ) participated in the 10-week longitudinal study. None of the participants reported any history of psychiatric, neurological, or pulmonary disorders, or use of medication that would affect the peripheral or central nervous system. All participants provided written informed consent before the experiment began and received US $\$ 20$ per session as a reward for their participation. The study was approved by the Institutional Review Committee of Chungnam National University (No. 201309-SB-004-01).

\section{Emotion-provoking stimuli}

We used film clips as emotional stimuli to provoke six basic emotions, since they are among the most effective stimuli to elicit affective responses in an experimental laboratory [25-28]. Film stimuli are dynamic media that provide visual and auditory input simultaneously, which contain more information relevant to the development of integrated and sustained emotional responses compared with still pictures (e.g., International Affective Picture System; IAPS), which elicit only short-lived affective responses [25-28]. Therefore, presenting film stimuli is an ecologically valid methodology to induce different affective states [25-28]. Additionally, they can evoke patterned psychophysiological responses, which may help to identify changes in the ANS during emotion elicitation [28].

A total of 60 film clips (10 clips per emotion) were excerpted from a variety of movies and TV programs, including documentaries and dramas (Table 1). Each clip was 2-4-min long. These film clips were used in our previous study and effectively induced the intended emotions in participants [29]. The stimuli were counterbalanced to minimize order effects.

\section{Psychological measurements}

Psychological responses of each participant were assessed by a self-rating questionnaire used in our previous study [29]. This includes three questions. First, participants were asked to label what specific emotion they had experienced during exposure to each stimulus. They selected one of seven choices: happiness, sadness, anger, fear, disgust, surprise, and not applicable. Second, participants evaluated the emotion's intensity, which reflected how strongly they felt the emotion during presentation of the stimulus, using an 11-point Likert scale, ranging from 1 (weakest) to 11 (strongest) [30]. Finally, the participants described the scene in which they felt the strongest emotion during exposure to each stimulus.

We used the results from the first and second questions as psychological responses. The appropriateness of the stimuli was evaluated by the first question, which reflects consistency between the intended emotion of the stimuli and the emotion experienced by the participants. This was assessed as the percentage of the participants who labeled the intended emotion correctly. The effectiveness of the stimuli was evaluated by the second question, which represents the intensity of emotion experienced by the participants for each stimulus. This used raw scores from the participants' ratings of each 
Table 1 Description of the emotion-provoking film clips

\begin{tabular}{ll}
\hline Emotion & Description of contents \\
\hline Happiness & Joyful scenes showing victories, weddings, or laughing \\
Sadness & Frustration or grief scenes showing death of a parent or partner \\
Anger & Deliberately harmful and unfair behaviors, such as a massacre or violent beating \\
Fear & Scary scenes showing a ghost or haunted house \\
Disgust & Disgusting scenes showing mutilation, butchery, or contamination \\
Surprise & Sudden or unexpected screaming scenes caused by a startling accident \\
\hline
\end{tabular}

emotion's intensity. The third question was used to determine the interval in the emotion-provoking phase, in which physiological data were analyzed (Fig. 1).

\section{Procedure}

The experiment was conducted in a soundproof room, which reduced noise by at least $35 \mathrm{~dB}$, to block outside noises or artifacts. Participants sat on a comfortable chair placed $2 \mathrm{~m}$ away from a 38-in TV monitor located in the center of the room. Prior to the experiment, electrodes for acquiring physiological signals were placed on participants' wrists, fingers, and ankle. Alcohol swabs and cotton pads were used to clean skin surfaces before attachment. The measurement began with a 60-s baseline phase, during which physiological signals were recorded and without any emotional stimulus (a blue screen presented without an auditory stimulus). Then, participants were presented with an emotion-provoking stimulus for 2-4 min. After the film clip presentation, they were asked to complete the three questions described above [28]. After the ratings, they were given 2 min to allow recovery of their emotional state. Then, the measurement started with a new baseline again. This cycle of baseline phase, emotion-provoking phase, selfratings, and recovery was repeated six times for each emotion. The order of stimuli was randomized for each participant. Total length of the experiment was $\sim 1.5 \mathrm{~h}$. Previous studies on emotion-specific ANS responses have used a separate neutral stimulus as a control condition. However, baseline measurement without any stimulus has also been used when a neutral stimulus is not favorable. We decided not to use a neutral film as a control since presenting a film clip itself can induce undesirable emotion elicitation in participants. Instead, we used a blue screen without any auditory stimulus during the baseline measurement.

The same experimental procedure was repeated 10 times on a weekly basis for each participant. To control the factors that could affect the baseline physiological responses, we contacted the participants by telephone a day before the scheduled experiment and instructed them to avoid smoking and consuming alcohol and

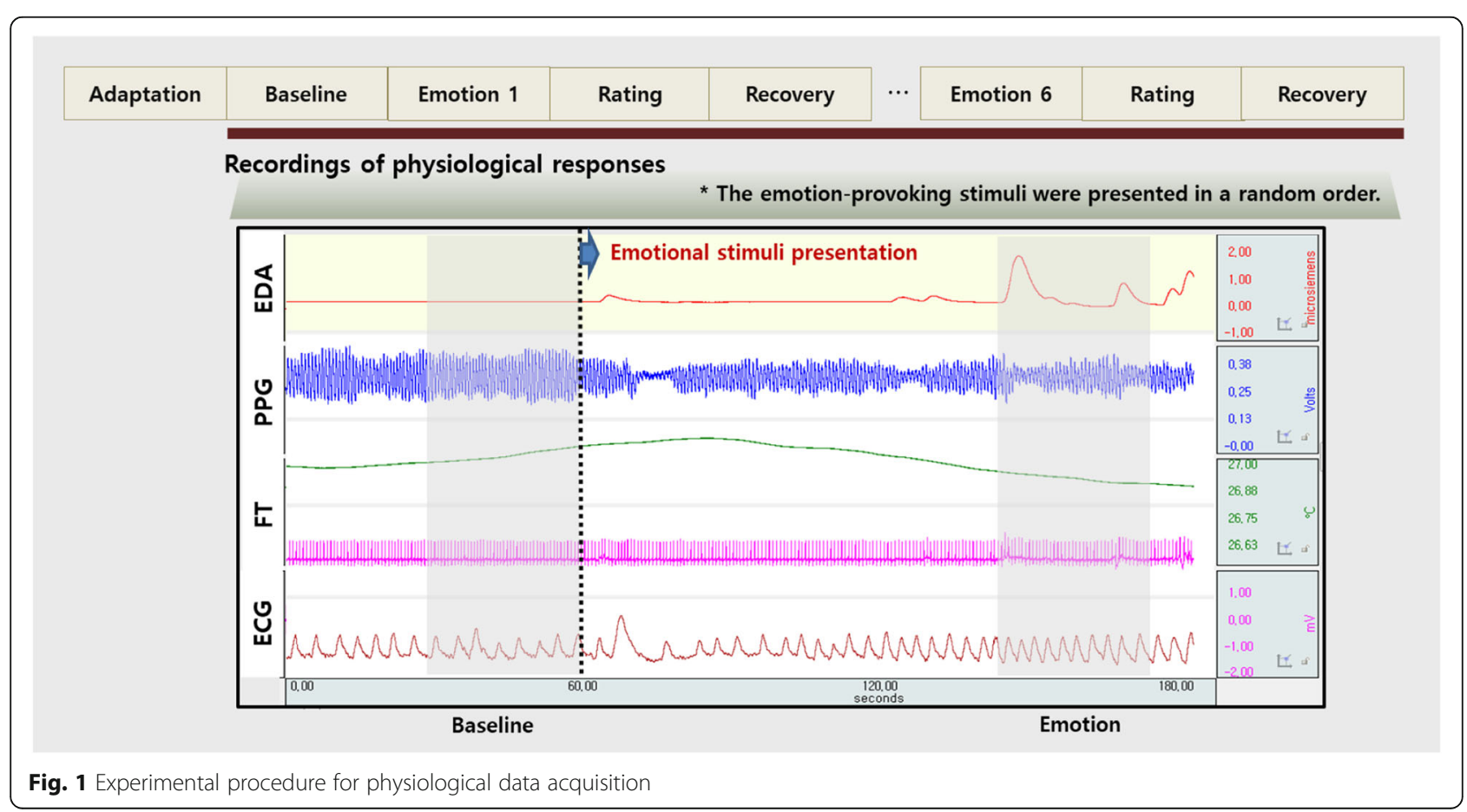


caffeine after $8 \mathrm{pm}$. They were also asked to get sufficient sleep. Furthermore, the experiment was repeated over 10 weeks at the same time during working hours $(10 \mathrm{am}-3 \mathrm{pm})$ and on the same day of the week for each participant. We changed film clips used for each emotion every week to avoid habituating participants by repeating the same stimulus. Therefore, 10 different film clips were prepared for each emotion, and a total of 60 film clips were used for 10 weeks.

\section{Physiological measurement}

We used the MP100WS system (Biopac Systems Inc., USA) to measure electrodermal activity (EDA), electrocardiogram (ECG), fingertip temperature (FT), and blood pulse volume (BVP). Display of recorded signals and data analysis were performed with AcqKnowledge software version 3.9.1 (Biopac Systems Inc., USA). An example of physiological data acquired before and during a fearful stimulus is shown in Fig. 1.

The EDA signal was recorded using an EDA100C amplifier, which applies a constant voltage $(0.5 \mathrm{~V})$ between two electrodes to measure skin conductance. The amplifier was connected to a set of TSD203 electrodes, which were attached to the palmar surfaces of the middle phalanges of the first and second fingers of the nondominant hand. The electrodes were filled with Electrode Paste EC33 (Grass Technologies, USA). Sampling rate was $200 \mathrm{~Hz}$. After movement and electrode contact artifacts removed, skin conductance level (SCL, in $\mu$ S) was calculated by averaging skin conductance signals for a 30-s interval that was determined by the self-rating questionnaire.

The ECG signal was recorded using an ECG100 amplifier, LEAD110S electrode leads, and EL503 electrodes. A lead I configuration was used for ECG measurement, in which three electrodes were attached to the bilateral wrists and left ankle. Sampling rate was $200 \mathrm{~Hz}$. Heart rate (HR, in beats per minute) was analyzed using AcqKnowledge, which detected R-waves in the ECG signals and calculated consecutive $\mathrm{R}-\mathrm{R}$ intervals. Finally, the mean HR (meanHR) was calculated by averaging $\mathrm{HR}$ values for the 30-s interval.

The FT signal was recorded using an FT100C amplifier and TSD202 temperature probe. The probe was attached to the volar surface of the distal phalanx of the little finger of the non-dominant hand. The mean FT (meanFT) was calculated by averaging FT values for the 30-s interval.

The BVP signal was recorded using a PPG100C amplifier and TSD200 transducer, which measured infrared reflectance of varying blood flow from the volar surface of the distal phalanx of the thumb of the non-dominant hand. Sampling rate was $200 \mathrm{~Hz}$. The mean BVP
(meanBVP) was calculated by averaging BVP signals for the 30-s interval.

As a result, total four physiological features, SCL, meanHR, meanFT, and meanBVP, were extracted from the measurement and used for data analysis.

\section{Data analysis}

All statistical analyses were performed using SPSS software version 18.0 (SPSS-IBM, Chicago, IL, USA). As psychological responses, the appropriateness and effectiveness of each emotion were evaluated by averaging 12 participants' ratings. To analyze physiological signals, a 30-s interval was selected from the total length for the baseline and each emotion-provoking phase. For the baseline, the final $30 \mathrm{~s}$ was used [29]. For the emotionprovoking phase, the interval was selected based on the participants' ratings. The participants described the scene in which they felt the strongest emotion during exposure to each stimulus. For example, a participant reported that she felt disgusted while watching the scene of a man putting his hand inside a dirty toilet, and we analyzed the $30-\mathrm{s}$ interval (26-55 s) containing this scene. Physiological features during this interval were evaluated as described above. We excluded the physiological data from participants who failed to elicit the targeted emotion during the analysis.

We adopted the most frequently used method for the measurement and analysis of emotion-specific ANS response. In particular, in most previous studies, the 30or 60-s interval immediately before the stimulus presentation has been selected as a baseline phase [4]. Other common intervals are 1/2-, 10-, 120-, 180-, and 300-s intervals. In addition, the averaging period did not influence the reported pattern of physiological responses [4], suggesting that a 30-s interval before the start of the stimulus can be considered as an appropriate baseline measurement. However, we cannot completely exclude the effect of anticipation of the stimuli on variations during the baseline.

We assessed the internal reliability of physiological responses observed during the 10 sessions, using Cronbach's alpha and intraclass correlation coefficient (ICC). Cronbach's alpha is widely used to evaluate internal consistency [31], which is defined as

$$
\alpha=\frac{k}{k-1}\left(1-\frac{\sum s_{i}^{2}}{s_{T}^{2}}\right)
$$

where $k$ is the number of sessions, $s_{i}^{2}$ is the variance of the $i$ th session, and $s_{T}^{2}$ is the variance of the total score formed by summing all the sessions. Furthermore, ICC is a widely used reliability index in test-retest and interrater reliability analyses [32-34], which assesses how 
strongly units in the same group resemble each other. The ICC coefficient is defined as

$$
\rho=\frac{\mathrm{MS}_{\mathrm{R}}-\mathrm{MS}_{\mathrm{E}}}{\mathrm{MS}_{\mathrm{R}}+(k-1) \mathrm{MS}_{\mathrm{E}}},
$$

where $M S_{R}$ is mean square for rows of raters or measurements, $\mathrm{MS}_{\mathrm{E}}$ is mean square for error, and $k$ is number of raters or measurements. ICC estimates and their 95\% confidence intervals were calculated based on the model of single rater/measurement, consistency, and 2way mixed-effects. Reliability indices less than 0.50 are indicative of poor reliability, values between 0.50 and 0.75 indicate moderate reliability, values between 0.75 and 0.90 indicate good reliability, and values greater than 0.90 indicate excellent reliability [32].

We compared physiological responses between the baseline and emotion-provoking phases using the Wilcoxon test since the Shapiro-Wilk test revealed that physiological features were not normally distributed. For this statistical test, we used all data ( 10 trials $\times 12$ subjects), except for data from participants who failed to elicit the targeted emotion, which were excluded in the calculation. Therefore, the amount of data used in the analysis was not identical for all conditions. For example, in the happiness condition, we evaluated data from 11 subjects for the eighth and tenth sessions because one subject failed to report the intended emotion during these sessions. For the remainder of the sessions, data from all 12 subjects were included in the calculation.

\section{Results}

Validity of psychological responses

Psychological responses to emotional stimuli were assessed by their appropriateness and effectiveness
(Tables 2 and 3). The mean appropriateness of each emotion ranged from 75 to $100 \%$, and the overall mean appropriateness from all the sessions and emotions was 93.3\% (Table 2). These results suggest that the film clips were valid stimuli for provoking the intended emotions. The mean appropriateness was higher in happiness, sadness, and disgust, compared with anger, fear, and surprise. The mean effectiveness of each emotion ranged from 8.4 to 10.8 points, and the overall mean effectiveness from all the sessions and emotions was 9.4 points (Table 3). These results also suggest that our stimuli effectively provoked strong emotions.

\section{Reliability of psychological responses}

We analyzed reliability of physiological responses to emotional stimuli using Cronbach's alpha and ICC. Reliability indices for the six emotions are summarized in Table 4 (Additional file 1: Tables S1-S6), which also present the descriptive statistics (mean and standard deviation) of physiological responses during the baseline and emotion-provoking phases. Cronbach's alphas from baseline measurement ranged from 0.13 to 0.79 , and those from emotion-provoking phases ranged from 0.39 to 0.96. Interestingly, when Cronbach's alphas from the baseline and emotion-provoking phases of the same physiological feature and same emotional stimulus were compared, Cronbach's alpha was always higher in the emotion-provoking phase than in the baseline, except meanFT during happiness. ICC values from the baseline measurements ranged from 0.10 to 0.70 , and those from emotion-provoking phases ranged from 0.38 to 0.96 . Similarly, ICC was always higher in the emotionprovoking phase than in the baseline of the same physiological feature and same emotional stimulus, except meanFT during happiness emotion. In particular,

Table 2 Mean appropriateness from participants' ratings

\begin{tabular}{|c|c|c|c|c|c|c|c|}
\hline \multirow[t]{2}{*}{ Session } & \multicolumn{6}{|l|}{ Emotion } & \multirow{2}{*}{$\begin{array}{l}\text { Mean } \\
(\%)\end{array}$} \\
\hline & HAP (\%) & SAD (\%) & ANG (\%) & FEA (\%) & DIS (\%) & SUR (\%) & \\
\hline 1 & 100 & 92 & 75 & 75 & 75 & 75 & 83 \\
\hline 2 & 100 & 100 & 75 & 100 & 92 & 92 & 94 \\
\hline 3 & 100 & 100 & 75 & 83 & 92 & 100 & 93 \\
\hline 4 & 100 & 100 & 75 & 92 & 100 & 100 & 95 \\
\hline 5 & 100 & 100 & 92 & 92 & 92 & 83 & 94 \\
\hline 6 & 100 & 100 & 92 & 92 & 100 & 83 & 95 \\
\hline 7 & 100 & 75 & 92 & 83 & 100 & 100 & 92 \\
\hline 8 & 92 & 100 & 83 & 100 & 83 & 83 & 92 \\
\hline 9 & 100 & 100 & 92 & 100 & 100 & 83 & 96 \\
\hline 10 & 92 & 100 & 92 & 75 & 100 & 75 & 91 \\
\hline Mean (\%) & 98 & 96 & 84 & 89 & 94 & 89 & 93.3 \\
\hline
\end{tabular}

$H A P$, happiness; SAD, sadness; $A N G$, anger; FEA, fear; DIS, disgust; SUR, surprise

Appropriateness was assessed as the percentage of the participants who labeled the intended emotion correctly 
Table 3 Mean effectiveness from participants' ratings

\begin{tabular}{|c|c|c|c|c|c|c|c|}
\hline \multirow[t]{2}{*}{ Session } & \multicolumn{6}{|l|}{ Emotion } & \multirow[t]{2}{*}{ Mean } \\
\hline & HAP & SAD & ANG & FEA & DIS & SUR & \\
\hline 1 & $8.4 \pm 1.07$ & $9.5 \pm 1.36$ & $9.7 \pm 1.88$ & $10 \pm 0.85$ & $10.2 \pm 0.95$ & $9.3 \pm 1.55$ & $9.5 \pm 1.28$ \\
\hline 2 & $8.9 \pm 1.30$ & $9.1 \pm 1.50$ & $9.9 \pm 1.07$ & $9.9 \pm 1.31$ & $10.8 \pm 1.08$ & $9.7 \pm 1.38$ & $9.6 \pm 1.27$ \\
\hline 3 & $8.8 \pm 1.31$ & $8.7 \pm 1.38$ & $9.7 \pm 1.54$ & $9.8 \pm 1.71$ & $9.9 \pm 1.34$ & $9.7 \pm 1.35$ & $9.3 \pm 1.44$ \\
\hline 4 & $9.6 \pm 1.17$ & $9.7 \pm 1.08$ & $9.5 \pm 1.16$ & $9.6 \pm 1.61$ & $10.4 \pm 1.04$ & $9.9 \pm 1.44$ & $9.7 \pm 1.25$ \\
\hline 5 & $9.6 \pm 1.24$ & $9.3 \pm 1.07$ & $9.8 \pm 1.68$ & $9.7 \pm 1.82$ & $9.7 \pm 1.27$ & $9.6 \pm 1.50$ & $9.6 \pm 1.43$ \\
\hline 6 & $9.3 \pm 1.49$ & $9.3 \pm 0.95$ & $9.4 \pm 1.47$ & $9.7 \pm 1.08$ & $10.3 \pm 0.93$ & $9.6 \pm 1.29$ & $9.5 \pm 1.20$ \\
\hline 7 & $9.3 \pm 1.23$ & $8.9 \pm 1.48$ & $8.9 \pm 1.31$ & $9.6 \pm 1.24$ & $9.3 \pm 1.31$ & $9.5 \pm 1.16$ & $9.3 \pm 1.29$ \\
\hline 8 & $8.4 \pm 1.34$ & $9.1 \pm 0.90$ & $9.2 \pm 1.07$ & $9.3 \pm 1.51$ & $10.2 \pm 0.97$ & $9.4 \pm 1.38$ & $9.2 \pm 1.20$ \\
\hline 9 & $9.7 \pm 1.51$ & $9.2 \pm 1.62$ & $9.5 \pm 1.82$ & $9.3 \pm 1.08$ & $10.1 \pm 1.21$ & $8.6 \pm 1.54$ & $9.4 \pm 1.46$ \\
\hline 10 & $8.8 \pm 1.42$ & $9.3 \pm 1.48$ & $9.7 \pm 1.07$ & $8.7 \pm 1.44$ & $10.1 \pm 1.16$ & $10.3 \pm 0.96$ & $9.5 \pm 1.26$ \\
\hline Mean & $9.1 \pm 1.31$ & $9.2 \pm 1.28$ & $9.5 \pm 1.41$ & $9.6 \pm 1.37$ & $10.1 \pm 1.13$ & $9.5 \pm 1.36$ & $9.4 \pm 1.31$ \\
\hline
\end{tabular}

$H A P$, happiness; SAD, sadness; $A N G$, anger; FEA, fear; DIS, disgust; SUR, surprise

Cronbach's alpha and ICC of SCL, meanHR, and meanBVP from emotion-provoking phases were higher than 0.90 for all emotional conditions.

Figure 2 shows group level comparisons of physiological features between the baseline and emotionprovoking phases. There were significant differences in the SCL between the two phases in all emotion conditions except for anger; the SCL significantly decreased in the happiness and sad conditions but increased in the fear, disgust, and surprise conditions. The meanFT significantly decreased compared with the baseline during disgust emotion. The meanHR significantly decreased during all emotion-provoking phases, except for happiness, compared with the baseline. The meanBVP significantly decreased during disgust and surprise emotions compared with the baseline.

Table 4 Physiological responses and their reliability indices from the baseline and emotion-provoking phases of all emotional conditions

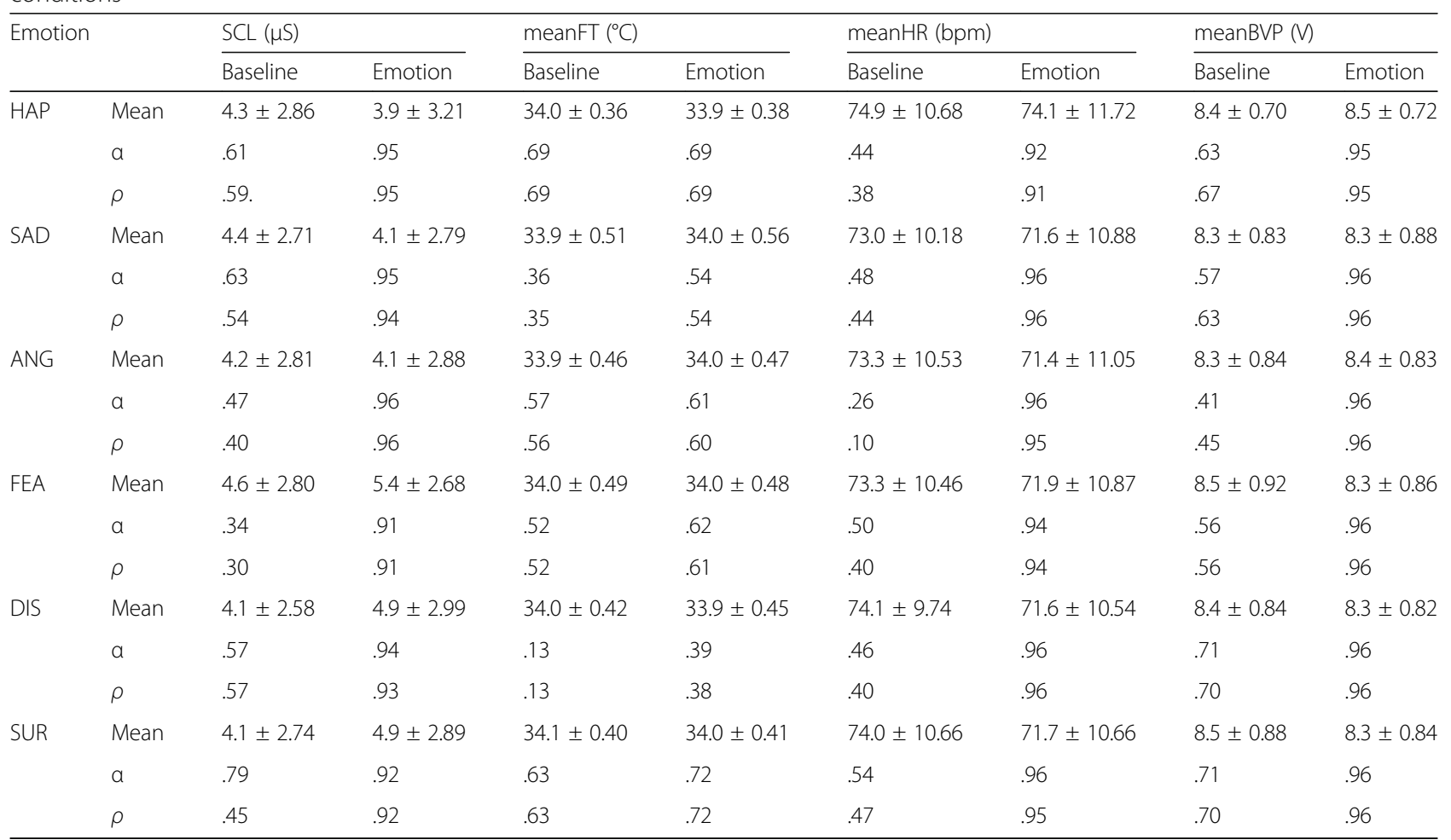




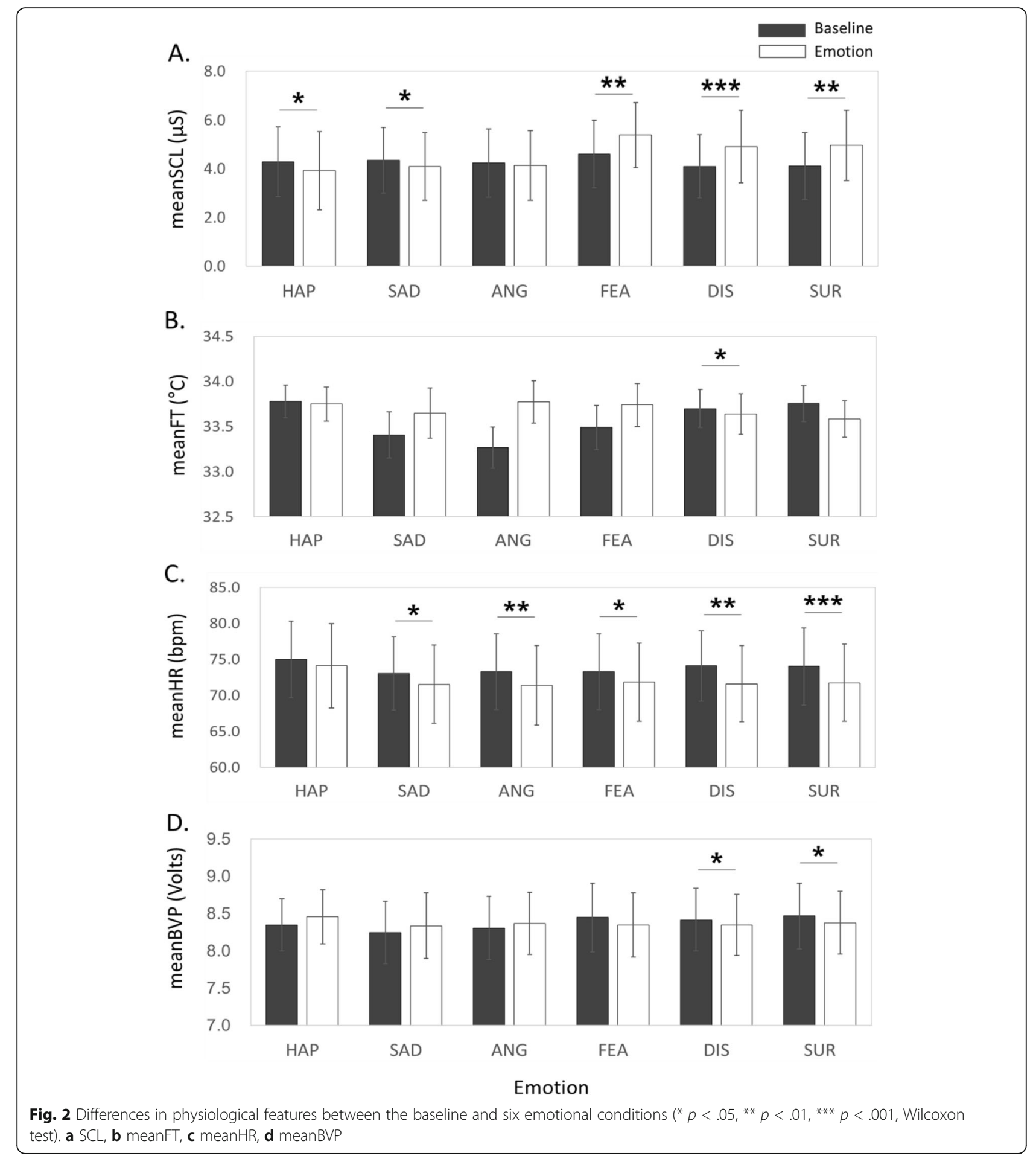

Figure 3 shows physiological features acquired from a single emotional condition (happiness) during 10 weekly sessions. Box plots in Fig. 3 compare the distributions of the features from the baseline and emotion-provoking phases of each subject. For the happiness condition, most subjects showed larger variations in the baseline than the emotion- provoking phase, except for the meanFT. As an example, Fig. 4 shows physiological features acquired from a single subject during 10 weekly sessions. Box plots in Fig. 4 compare the distributions of the features from the baseline and emotion-provoking phases of each emotional condition. For all six emotions, this subject showed larger variations in the 


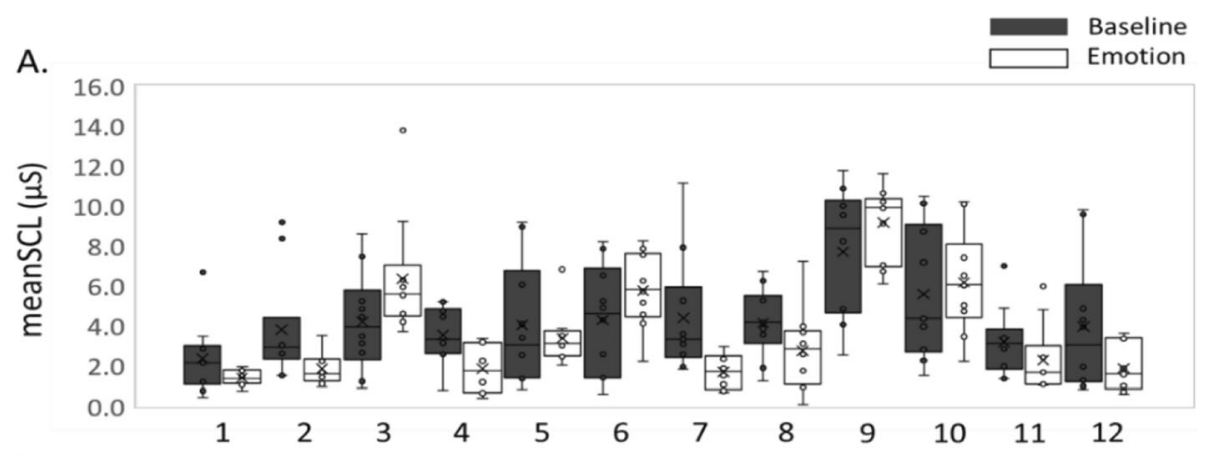

B.

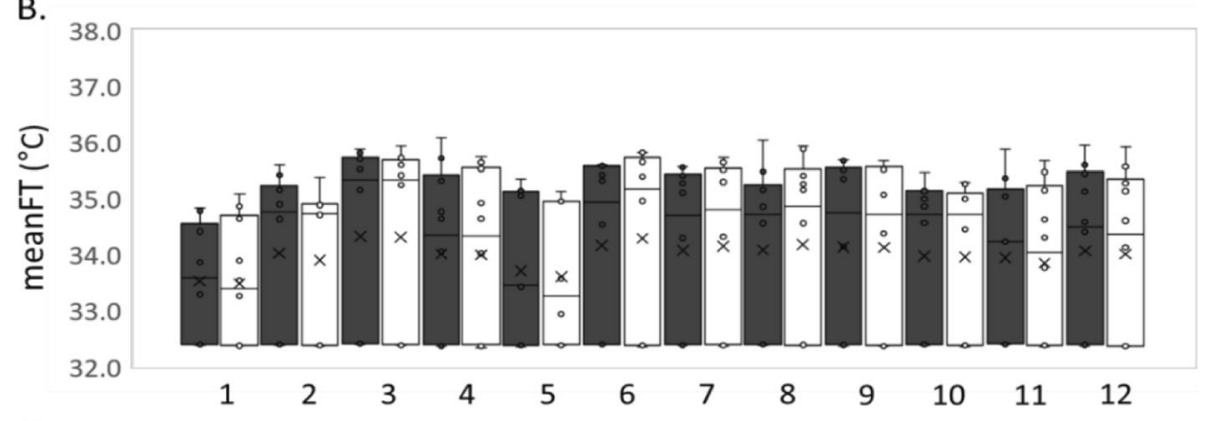

c.

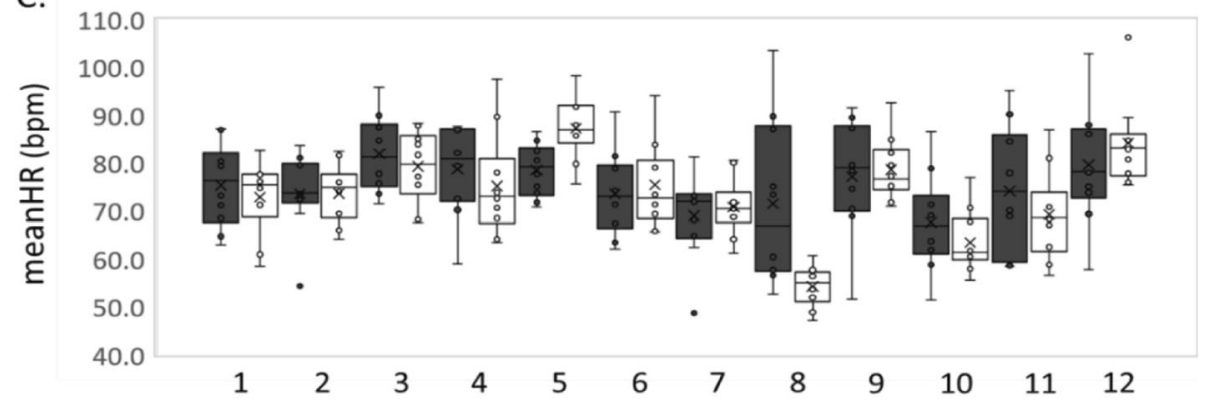

D.

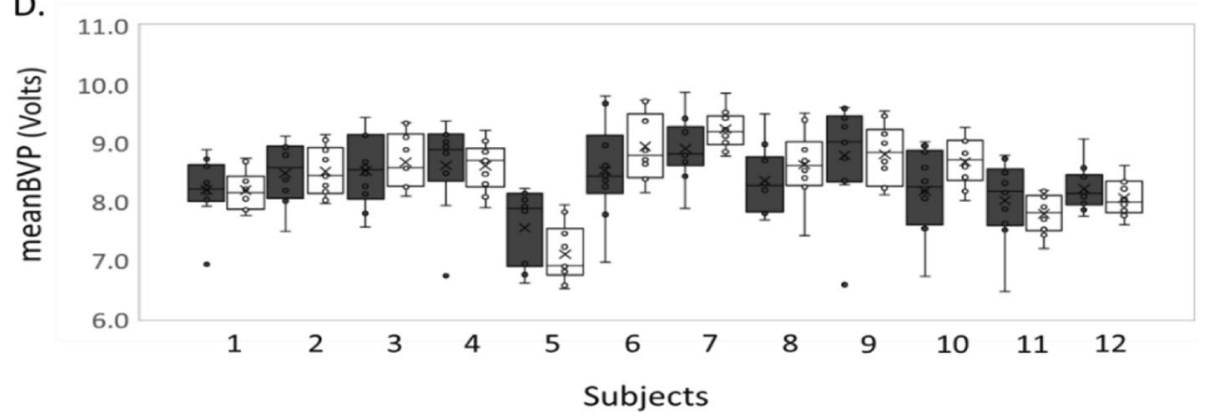

Fig. 3 Physiological features acquired from a single emotional condition (happiness) during 10 weekly sessions. Distributions of the features from the baseline and emotion-provoking phases of each subject are compared by the box plots. a SCL, $\mathbf{b}$ meanFT, c meanHR, $\mathbf{d}$ meanBVP

baseline than the emotion-provoking phase, except for the meanFT.

\section{Discussion}

We investigated the reliability of physiological responses induced by emotional stimuli for provoking basic emotions during 10 weekly sessions. The film clips used as emotional stimuli in the present study were confirmed to be appropriate and effective in inducing the intended emotions regardless of the variations in film clips (Tables 2 and 3). Film clips can produce more active and vivid emotional responses than static emotional stimuli (e.g., facial expressions, slides, and imagery) and can be considered advantageous in terms of ecological validity, to the extent that emotions are induced by dynamic visual and auditory stimuli $[35,36]$. 


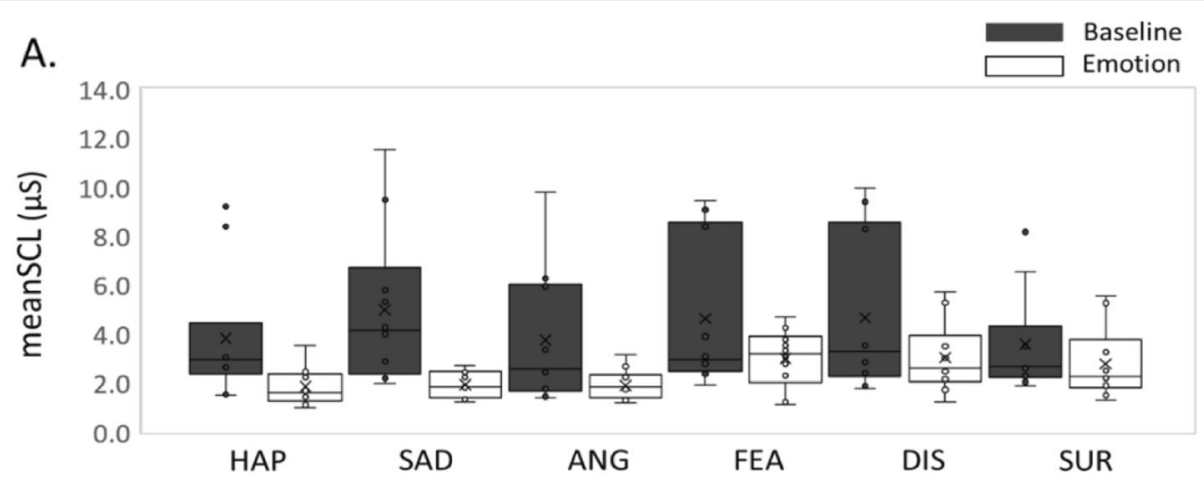

B.

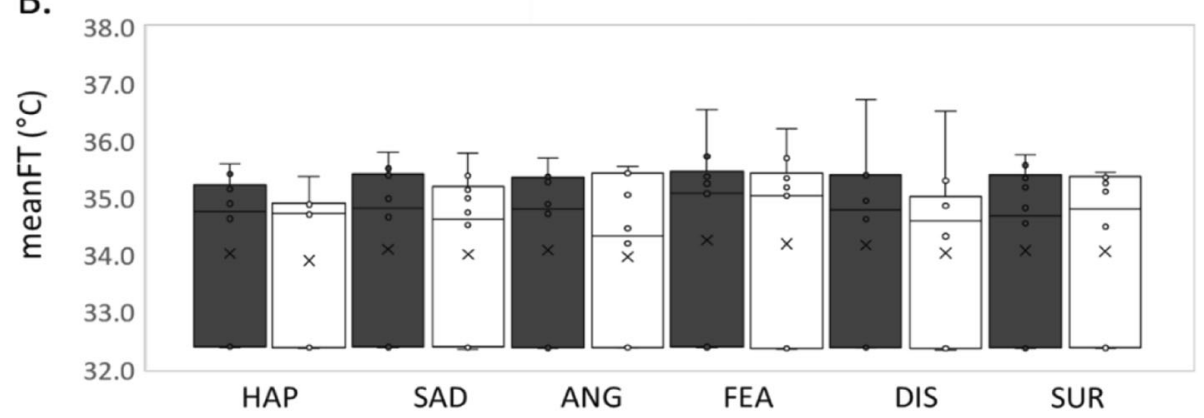

C.

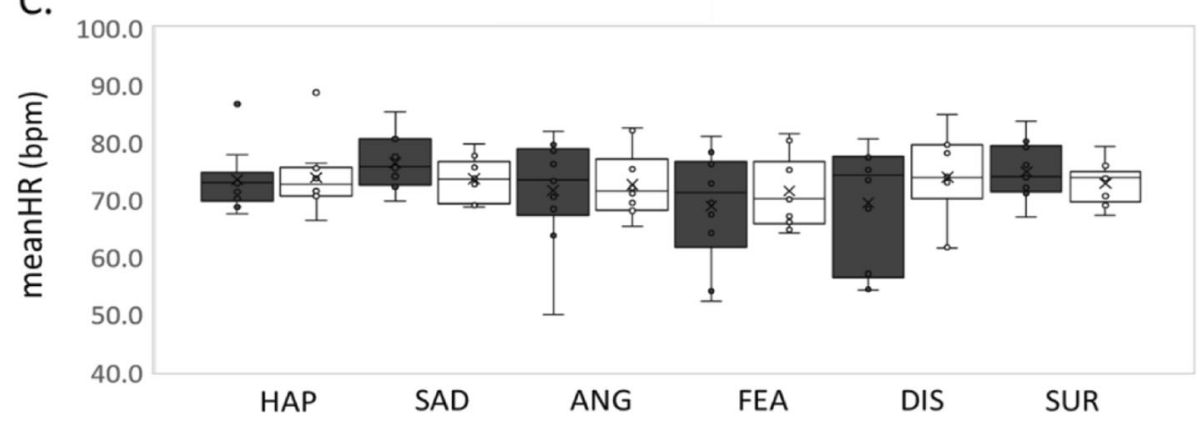

D.

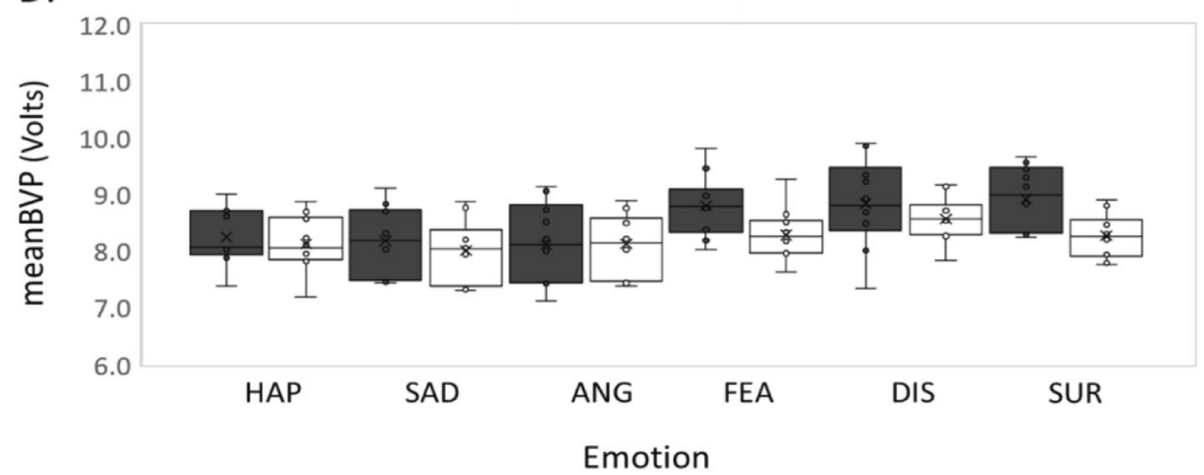

Fig. 4 Physiological features acquired from a single subject during 10 weekly sessions. Distributions of the features from the baseline and emotion-provoking phases of each emotional condition are compared by the box plots. a SCL, b meanFT, $\mathbf{c}$ meanHR, $\mathbf{d}$ meanBVP

However, film clips may also evoke other unanticipated emotions. For example, the mean appropriateness of anger, fear, disgust, and surprise stimuli for the first session was $75 \%$ (Table 2), indicating that some participants did not select the intended emotions from these stimuli as their experienced emotions. In fact, these results may support Levenson's [37] claim that emotions are far more complex in that people do not 
experience only one emotion during a particular situation; instead, they experience several mixed emotions that have dominance over one another in a relatively consistent pattern. Christie and Friedman [36] have applied pattern classification analyses to discriminate seven distinct emotions experienced by subjects based on an 18-item affect self-report scale. In this study, the sadness condition was predicted correctly for 27 out of 34 subjects, whereas the remaining seven subjects were classified as experiencing the amusement, anger, contentment, and disgust conditions [36]. Similarly, when disgust was expected, 15 out of 34 subjects were classified as experiencing amusement, anger, fear, and sadness [36]. These results suggest that it is difficult to elicit a single discrete emotion using any given stimulus, yet it is possible to induce most commonly experienced emotions. Therefore, it may be realistic to aim to acquire the most frequently reported emotion from a given stimulus rather than to expect a perfect match between the intended and experienced emotions. This may partially explain why we observed large variations in individual responses.

We observed that the emotional stimuli significantly affected SCL and meanHR, which are the most frequently reported measures for sympathetic and parasympathetic autonomic activity, suggesting that these two features were indeed strong indicators of ANS responses (Fig. 2). Our results were also consistent with previous studies on physiological responses to emotional stimuli; for example, all emotions, except for contentment, noncrying sadness, and acute sadness, induced an increase in SCL; facial expressions of anger induced decreases in HR and SCL; HR decreased during contentment, noncrying and acute sadness, imminent-threat fear, and mutilation-related disgust conditions [4].

Both reliability indices, Cronbach's alpha and ICC, were lower in the baseline than in the emotionprovoking phase of all emotions. In particular, Cronbach's alpha and ICC in the baseline phase ranged from 0.13 to 0.79 and from 0.10 to 0.70 , respectively, indicating poor or moderate reliability. These results suggest that intra- and interindividual variations in the baseline level were relatively large. Since an individual's initial baseline level will affect the degree to which his/her autonomic system responds to emotional stimuli, it is important to assess the baseline and incorporate this information for interpreting physiological changes in response to the emotional stimulus [38]. For example, the differential values between the baseline and emotional states can be used for statistical tests or the baseline can be used as a covariate in analysis of covariance for physiological response during the emotional state.

In contrast, Cronbach's alpha and ICC of SCL, meanHR, and meanBVP in the emotion-provoking phase both ranged from 0.91 to 0.96 , indicating excellent reliability [32]. Therefore, SCL, meanHR, and meanBVP measured from the emotion-provoking phase exhibited excellent internal consistency and reliability throughout the 10 weekly sessions $[39,40]$. However, the reliability indices of meanFT during the emotion-provoking phase ranged from 0.38 to 0.72 , indicating poor or moderate reliability. In particular, meanFT in disgust showed the lowest reliability (Tables 4 and Additional file 1: Table S5). Similarly, the change in FT in response to disgust stimuli is known to be inconsistent [4]. For example, disgust elicited in relation to mutilation induced consistent increases in HR and SCL but mixed results in FT [4]. Furthermore, the FT response depended on the type of disgust stimuli; FT was unaffected by personalized recall [41], increased by directed facial action or personalized recall [42-44], and decreased by picture or film clip presentations [45-48]. In our previous study [29], the same film stimuli for disgust used in the present study exhibited substantially high appropriateness and effectiveness, and we expected that the disgust stimuli would lead to reliable physiological responses in subjects. Nonetheless, the meanFT during the emotion-provoking phase was not consistent throughout the 10 sessions.

\section{Limitations}

We used 10 different film clips for each emotion to avoid adaptation of participants caused by repeating the same stimulus. However, different contexts designed to elicit the same emotion can lead to different physiological responses. For example, disgust related to contamination and pollution is accompanied by sympathetic-parasympathetic co-activation, whereas disgust related to mutilation, injury, and blood is accompanied by sympathetic deactivation and unchanged vagal activation [4]. We did not investigate the effect of different contexts of the film stimuli for the same targeted emotion on physiological responses. Therefore, to evaluate the reliability more accurately, we may need to subdivide the film stimuli with respect to their contexts.

During the baseline measurement, we did not use a neutral film stimulus because presenting a film clip itself can induce undesirable emotion elicitation. Instead, we used a blue screen without any auditory stimulus. In a future study, we may need to include neutral film clips to evaluate the reliability of physiological responses to the neutral condition.

Participants were given 2 min to allow recovery between emotional conditions. However, a 2-min recovery period may not be sufficient to fully recover from emotional states, especially after a negative emotion inducement (e.g., anger). This may partially explain why we observed large variations in baseline measurement. 


\section{Conclusions}

In conclusion, we evaluated the inter- and intraindividual reliability of physiological responses induced by emotional stimuli during 10 weekly sessions, using nonidentical film clips as stimuli. We hypothesized that physiological responses would show high consistency in both the baseline and emotional phases. However, intraand interindividual variations in the baseline level were relatively large. We demonstrated excellent internal reliability and intraclass consistency in SCL, meanHR, and meanBVP during the emotion-provoking phase throughout the 10 sessions, suggesting that these features can be used as reliable physiological indices in emotion studies. Additionally, our findings can be applied to various emotion-related research fields, such as emotion recognition, development of emotion theory, and profiling emotion-specific physiological responses.

\section{Supplementary information}

Supplementary information accompanies this paper at https://doi.org/10. 1186/s40101-019-0209-y.

Additional file 1: Table S1. Physiological responses and their reliability indices from the baseline and emotion-provoking phases of the happiness condition. Table S2. Physiological responses and their reliability indices from the baseline and emotion-provoking phases of the sadness condition. Table S3. Physiological responses and their reliability indices from the baseline and emotion-provoking phases of the anger condition. Table S4. Physiological responses and their reliability indices from the baseline and emotion-provoking phases of the fear condition. Table S5 Physiological responses and their reliability indices from the baseline and emotion-provoking phases of the disgust condition. Table S6. Physiological responses and their reliability indices from the baseline and emotion-provoking phases of the surprise condition.

\section{Abbreviations}

ANS: Autonomic nervous system; BVP: Blood volume pulse; ECG: Electrocardiogram; EDA: Electrodermal activity; FT: Fingertip temperature; HR: Heart rate; ICC: Intraclass correlation coefficient; SCL: Skin conductance level

\section{Acknowledgements}

Not applicable.

\section{Authors' contributions \\ EHJ and JHS designed research and performed research. EHJ and MSP analyzed the data. EHJ, and SB wrote the paper. All authors commented on the manuscript. All authors agreed to this submission. The material is an original research and has not been previously published while under consideration.}

\section{Funding}

This work was supported by the Institute for Information \& Communications Technology Promotion (IITP) grant funded by the Korea government (MSIT) (No. 2015-0-00062). This research was also supported by Basic Science Research Program of the National Research Foundation of Korea (NRF) funded by the Ministry of Education (NRF-2015R1D1A1A01059095) and the NRF grant funded by the MSIT (No. 2017R1C1B5017730).

Availability of data and materials

Not applicable

\section{Ethics approval and consent to participate}

This study was approved by the ethics committee of the Institutional Review Board of the Chungnam National University (No. 201309-SB-004-01). All participants provided written consent for their participation after they were fully informed about the study and received US\$20 per session as a reward for their participation

\section{Consent for publication}

Not applicable.

\section{Competing interests}

The authors declare that they have no competing interests.

\section{Author details}

${ }^{1}$ Welfare \& Medical ICT Research Department, Electronics and Telecommunications Research Institute (ETRI), 218 Gajeong-ro, Yuseong-gu, Daejeon 34129, Republic of Korea. ${ }^{2}$ Department of Electronics Engineering, Incheon National University, 119 Academy-ro, Yeonsu-gu, Incheon, Republic of Korea. ${ }^{3}$ Department of Rehabilitation Counselling, Seoul Hanyoung University, 290-42 Kyoungin-ro, Guro-gu, Seoul 08274, Republic of Korea. ${ }^{4}$ Department of Psychology, Brain Research Institute, Chungnam National University, 99 Daehak-ro, Yuseong-gu, Daejeon 34134, Republic of Korea.

Received: 14 May 2019 Accepted: 28 October 2019

Published online: 28 November 2019

\section{References}

1. Steimer T. The biology of fear- and anxiety-related behaviours. Dialogues Clin Neurosci. 2002;4:231-49.

2. Cannon WB. Bodily changes in pain, hunger, fear, and rage. New York: Appleton \& Company; 1915.

3. Brown TM, Fee E. Walter Bradford Cannon: pioneer physiologist of human emotions. Am J Public Health Res. 2002;92:1594-5.

4. Kreibig SD. Autonomic nervous system activity in emotion: A review. Biol Psychol. 2010;84:394-421.

5. Cacioppo JT, Berntson GG, Klein DJ, Poehlmann KM. Psychophysiology of emotion across the lifespan. Annu Rev Gerontol. 1997;17:27-74.

6. Cacioppo JT, Berntson GG, Larsen JT, Poehlmann KM, Ito TA. The psychophysiology of emotion. In: Lewis R, Haviland-Jones JM, editors. The handbook of emotion. 2nd ed. New York: Guilford Press; 2000.

7. Hinz A, Hueber B, Schreinicke O, Seibt R. Temporal stability of psychophysiological response patterns: concepts and statistical tools. Int J Psychophysiol. 2002;44:57-65.

8. Lacey Jl, Lacey BC. The law of initial value in the longitudinal study of autonomic constitution: reproducibility of the autonomic responses and response patterns over a four years interval. Ann N Y Acad Sci. 1962;98: 1257-90.

9. Fredrikson M, Danielssons T, Engel BT, Frisk-Holmberg M, Strom G, Sundin O. Autonomic nervous system function and essential hypertension: individual response specificity with and without beta-adrenergic blockade. Psychophysiology. 1985;22:167-74.

10. Robinson JW, Whitsett SF, Kaplan BJ. The stability of physiological reactivity over multiple sessions. Biol Psychol. 1987;24:129-39.

11. Waters WF, Williamson DA, Bernard BA, Blouin DC, Faulstich ME. Test-retest reliability of psychophysiological assessment. Behav Res Ther. 1987;25:21321.

12. Arena JG, Goldberg SJ, Saul DL, Hobbs SH. Temporal stability of psychophysiological response profiles: Analysis of individual response stereotypy and stimulus specificity. Behaviour Therapy. 1989;20:609-18.

13. Marwitz M, Stemmler G. On the status of individual response specificity. Psychophysiology. 1998;35:1-15.

14. Burgess AP, Gruzelier JH. The reliability of event-related desynchronisation: a generalisability study analysis. Int J Psychophysiol. 1996;23:163-9.

15. Krause CM, Sillanmäki L, Häggqvist A, Heino R. Test-retest consistency of the event-related desynchronization/event-related synchronization of the 4-6, 68, 8-10 and $10-12 \mathrm{~Hz}$ frequency bands during a memory task. Clin Neurophysiol. 2001;112:750-7.

16. Manber R, Allen JJB, Burton K, Kaszniak AW. Valence-dependent modulation of psychophysi-ological measures: Is there consistency across repeated testing. Psychophysiology. 2000;37:683-92. 
17. Larson CL, Ruffalo D, Nietert JY, Davidson RJ. Temporal stability of the emotion-modulated startle response. Psychophysiology. 2000;37:92-101.

18. Bradley MM, Gianaros P, Lang P. As time goes by: Stability of startle modulation. SPR abstracts, S21; 1995.

19. Doussard-Roosevelt JA, Montgomery LA, Porges SW. Short-term stability of physiological measures in kindergarten children: respiratory sinus arrhythmia, heart period, and cortisol. Dev Psychobiol. 2003;43:230-42.

20. Stephens $\mathrm{CL}$, Christie IC, Friedman BH. Autonomic specificity of basic emotions: Evidence from pattern classification and cluster analysis. Biol. Psychol. 2010;84:463-73.

21. Fishel SR, Muth ER, Hoover AW. Establishing appropriate physiological baseline procedures for real-time physiological measurement. J Cogn Eng Decis Making. 2007;1:286-308.

22. Kistler A, Mariauzouls C, von Berlepsch K. Fingertip temperature as an indicator for sympathetic responses. Int J Psychophysiol. 1998;29:45-57.

23. Kamal AA, Harness JB, Irving G. Skin photoplethysmography-a review. Comput Methods Programs Biomed. 1989;28:257-69.

24. Allen J. Photoplethysmography and its application in clinical physiological measurement. Physiol Meas. 2007;28:R1-R39.

25. Bos MGN, Jentgens $P$, Beckers T, Kindt M. Psychophysiological response patterns to affective film stimuli. Plos One. 2013;8:e622661.

26. Lazarus RS, Speisman JC, Mordkoff AM, Davidson LA. A laboratory study of psychological stress produced by an emotion picture film. Psychol Monogr. 1962;76:553.

27. Davis MH, Hull JG, Young RD, Warren GG. Emotional reactions to dramatic film stimuli: the influence of cognitive and emotional empathy. J Pers Soc Psychol. 1987;52:126-33.

28. Palomba D, Sarlo M, Angrilli A, Mini A, Stegagno L. Cardiac responses associated with affective processing of unpleasant film stimulus. Int J Psychophysiol. 2000;36:45-57.

29. Park BJ, Jang EH, Chung MA, Kim SH. Design of prototype-based emotion recognizer using physiological signals. ETRI Journal. 2013;35:869-79.

30. Likert R. A technique for the measurement of attitudes. Arch of Psychol. 1932;140:44-53.

31. Cronbach $\sqcup$. Coefficient alpha and the internal structure of tests. Psychometrika. 1951;16:297-334.

32. Koo TK, Li MY. A Guideline of Selecting and Reporting Intraclass Correlation Coefficients for Reliability Research. J Chiropr Med. 2016;15:155-63.

33. McGraw KO, Wong SP. Forming inferences about some intraclass correlation coefficients. Psychol Methods. 1996;1:30-46.

34. Shrout PE, Fleiss JL. Intraclass correlations: uses in assessing rater reliability. Psychol Bull. 1979;86:420-8.

35. Gross JJ, Levenson RW. Emotion elicitation using films. Cogn Emot. 1995;9: 87-108.

36. Christie I, Friedman B. Autonomic specificity of discrete emotion and dimensions of affective space: A multivariate approach. Int J Psychophysiol. 2004; $51: 143-53$

37. Levenson RW. Human emotion: a functional view. In: Ekman P, Davidson RJ, editors. The nature of emotion: Fundamental questions. New York: Oxford University Press; 1994. p. 123-6.

38. Cassidy J, Shaver PR. Handbook of attachment (3rd ed.): theory, research, and clinical applications. In: Hane AA, Fox NA, editors. Studying the biology of human attachment. New York: Guilford Press; 2016.

39. Kline P. The handbook of psychological testing. 2nd ed. London: Routledge; 2000.

40. George D, Mallery P. SPSS for Windows step by step: a simple guide and reference. 11.0 update. 4th ed. Boston: Allyn \& Bacon; 2003.

41. Prkachin K, Williams-Avery R, Zwaal C, Mills D. Cardiovascular changes during induced emotion: an application of Lang's theory of emotional imagery. J Psychosom Res. 1999;47:255-67.

42. Ekman P, Levenson RW, Friesen W. Autonomic nervous system activity distinguishes among emotions. Science. 1983;221:1208-10.

43. Levenson RW, Ekman P, Friesen W. Voluntary facial action generates emotion-specific autonomic nervous system activity. Psychophysiology. 1990;27:363-84.

44. Levenson RW, Ekman P, Heider K, Friesen W. Emotion and autonomic nervous system activity in the Minangkabau of West Sumatra. J Pers Soc Psychol. 1992;62:972-88.

45. Collet C, Vernet-Maury E, Delhomme G, Dittmar A. Autonomic nervous system response patterns specificity to basic emotions. J Auton Nerv Syst. 1997;62:45-57.
46. Gross JJ. Antecedent- and response-focused emotion regulation: divergent consequences for experience, expression, and physiology. J Pers Soc Psychol. 1998;74:224-37.

47. Gross JJ, Levenson RW. Emotional suppression: physiology, self-report, and expressive behavior. J Pers Soc Psychol. 1993;64:970-86.

48. Levenson RW, Carstensen LL, Friesen WV, Ekman P. Emotion, physiology, and expression in old age. Psychol Aging. 1991;6:28-35.

\section{Publisher's Note}

Springer Nature remains neutral with regard to jurisdictional claims in published maps and institutional affiliations.
Ready to submit your research? Choose BMC and benefit from:

- fast, convenient online submission

- thorough peer review by experienced researchers in your field

- rapid publication on acceptance

- support for research data, including large and complex data types

- gold Open Access which fosters wider collaboration and increased citations

- maximum visibility for your research: over $100 \mathrm{M}$ website views per year

At BMC, research is always in progress.

Learn more biomedcentral.com/submissions 